\title{
Effects of Linewidth Broadening Method on Recoil of Sodium Laser Guide Star
}

\author{
Xiangyuan Liu ${ }^{1,2, *}$, Xianmei Qian ${ }^{3}$, Rui He ${ }^{1}$, Dandan Liu ${ }^{1} \mathbb{D}$, Chaolong Cui ${ }^{3}$, Chuanyu Fan ${ }^{1}$ and Hao Yuan ${ }^{1} \mathbb{D}$ \\ 1 School of Electrical and Photoelectronic Engineering, West Anhui University, Lu'an 237012, China; \\ heruim@wxc.edu.cn (R.H.); dandan86@mail.ustc.edu.cn (D.L.); fcy@mail.ustc.edu.cn (C.F.); \\ yuanhao@wxc.edu.cn (H.Y.) \\ 2 State Key Laboratory of Pulsed Power Laser Technology, School of Electronic Countermeasures, \\ National University of Defense Technology, Hefei 230031, China \\ 3 Anhui Institute of Optics and Fine Mechanics, Chinese Academy of Sciences, Hefei 230031, China; \\ qianxianmei@aiofm.ac.cn (X.Q.); clcui@aiofm.ac.cn (C.C.) \\ * Correspondence: 1xy0564@aliyun.com; Tel.: +86-15955915022
}

check for updates

Citation: Liu, X.; Qian, X.; He, R.; Liu, D.; Cui, C.; Fan, C.; Yuan, H. Effects of Linewidth Broadening Method on Recoil of Sodium Laser Guide Star. Atmosphere 2021, 12, 1315. https://doi.org/10.3390/ atmos12101315

Academic Editors: Nataliya V. Bakhmetieva and Gennadiy I. Grigoriev

Received: 17 August 2021 Accepted: 29 September 2021 Published: 8 October 2021

Publisher's Note: MDPI stays neutral with regard to jurisdictional claims in published maps and institutional affiliations.

Copyright: (c) 2021 by the authors. Licensee MDPI, Basel, Switzerland. This article is an open access article distributed under the terms and conditions of the Creative Commons Attribution (CC BY) license (https:/ / creativecommons.org/licenses/by/ $4.0 /)$.

\begin{abstract}
The linewidth broadening of the circular-polarized continuous wave laser mitigates the recoil effects of the sodium laser guide star very well. By choice of the optimal laser linewidth, the relations between the laser intensity and average spontaneous emission rates are obtained. The numerically simulated results show that the 1-100 MHz linewidth broadening effectively weakens recoil and enhances the average spontaneous emission rates. For laser powers from $10 \mathrm{~W}$ to $60 \mathrm{~W}$, considering the intensity distribution with random at the mesospheric sodium layer, when the laser linewidth is broadened to be 1-100 MHz from $0 \mathrm{MHz}$, increments of the return photons go up to $110 \%$ from $50 \%$ and do not have an effect on the spot sizes of the sodium laser guide star. Several cases have proven that the linewidth broadening method is correct. Further calculations show that the linewidth broadening method similarly applies to the case of the multi-mode laser. Furthermore, the linewidth broadening of re-pumping should be taken into account.
\end{abstract}

Keywords: recoil effects; linewidth broadening; average spontaneous emission rate; return photons; mesospheric sodium layer

\section{Introduction}

There is $600 \mathrm{~kg}$ of sodium in the atmosphere all around the earth, which provides a fundamental condition for the form of the sodium laser guide star [1]. It is well known that the sodium laser guide star is applied in the wave-front detection of adaptive optics and helps to improve high resolution imaging through the atmosphere. The high return photons are beneficial to enhancing the signal to noise ratio in the wave-front detection. When the laser interacts with mesospheric sodium atoms, the three evils named by Holzlöhner [2] cause the drop in the brightness of the sodium laser guide star. The geomagnetic field effects weaken the polarization of the circular-polarized laser. The stimulated emission caused by a high intensity reduces the spontaneous radiation of sodium fluorescence. Recoil makes sodium atoms in the range illuminated by the laser drift to a higher and higher frequency as time goes on [3]. The recoil phenomenon leads to a decrease in the total number of excited sodium atoms and a reduction in spontaneous emission rates. Fortunately, the laser with $1.713 \mathrm{GHz}$ sidebands has been employed to pump sodium atoms from $\mathrm{F}=1$ to $\mathrm{F}=2$ ground states [4]. The continuous wave laser with limited powers enables the stimulated emission to be effectively dropped [5]. Not long ago, Bustos et al. [6] proposed a blue-drift method to drop recoil effects for the $0 \mathrm{MHz}$ linewidth laser. This work shows a $60 \%$ increase in return photons and a $50 \%$ decrease in re-pumping power for the sodium laser guide star excited by a continuous wave laser. In this article, we focus on the study of the linewidth broadening of the circular-polarized laser to alleviate 
recoil effects of the sodium laser guide star. In fact, the linewidth broadening method is used to modulate the intensity distribution of the laser in the spectrum to weaken recoil effects. The linewidth of the continuous wave laser is always less than $1 \mathrm{MHz}$, which is theoretically regarded as $0 \mathrm{MHz}$. We anticipate that there is a laser modulation technology that will be applied to broaden the laser linewidth from $0 \mathrm{MHz}$ or tens of $\mathrm{kHz}$ to above $1 \mathrm{MHz}$. Up to now, Chamoun and Digonnet [7] have proposed a Gaussian white noise (GWN) phase modulation to broaden the laser linewidth which uses the linear Pockels effect of the electro-optic crystal by an external electric field. Its advantages lie in that the laser wavelength can remain stable, and the modulated laser linewidth is independent of the natural linewidth of the laser. By the theoretical models and the numerical simulations, the return photons from the sodium laser guide star excited by the continuous wave (CW) laser are calculated. The study results show that the linewidth broadening method markedly increases the return photons. In the process of the research, theoretical models are presented in Section 2. The steady state solution of the two-level Bloch equation is applied in the excitation probability of sodium atoms. The average spontaneous emission rate of excited sodium atoms is introduced. The expressions of return photons and spot sizes of the sodium laser guide star are described. In Section 3, the numerical method is described in detail and the parameters are listed. In Section 4, results of the numerical simulations are obtained. Powerful evidence indicates that linewidth broadening can effectively weaken the recoil effects. Then, the choice of the optimal linewidth broadening is analyzed, and relational expressions between laser intensity and average spontaneous emission rates are obtained. In Section 5, we discuss the effects of linewidth broadening on the return photons and spot sizes of the sodium laser guide star and consider the linewidth broadening for the re-pumping laser. In particular, we further simulate the influence of linewidth broadening on the recoil effects of the sodium laser guide star in the case of the multi-mode laser. Finally, we summarize our work, including the optimal range of laser linewidth broadening and the impact on the returned photons from the sodium laser guide star.

\section{Theoretical Models}

The excitation of the sodium laser guide star needs to launch the sodium laser to the mesospheric sodium layer. Under the usual conditions, the spectrum center of the laser is required to aim at the center frequency of the Doppler shifts of the sodium atom under the thermal equilibrium states. The single-mode (mono-mode) CW laser is applied in the sodium laser guide star and has the highest intensity in the spectrum center. A great deal of sodium atoms move to a higher Doppler shift after absorbing laser photons, and the Doppler shift increases $50 \mathrm{kHz}$. This process can be described as follows [8]:

$$
\begin{aligned}
v_{D}^{\prime} & = \pm v_{D}+h /\left(\lambda^{2} m_{N a}\right) \\
& = \pm v_{D}+50 \mathrm{kHz}
\end{aligned}
$$

where $v_{D}^{\prime}$ is the Doppler shift after a sodium atom absorbs a photon, $v_{D}$ is the former Doppler shift, $h$ is the Plank constant, $\lambda$ is the $589.159 \mathrm{~nm}$ wavelengths, and $m_{\mathrm{Na}}$ is the mass of a sodium atom. " + " denotes the motion of a sodium atom along with the direction of laser propagation, and "-" denotes the opposite one. We consider the situation of two energy levels for interactions between the circularly polarized laser and sodium atoms. This process is described by the optical Bloch equation. The relevant theory and experiments $[9,10]$ have proven that when the circular-polarized light with wavelength $589.159 \mathrm{~nm}$ and sodium atoms with the $D_{2}$ structure interplay, in a very short time, all energy level transitions will come into the cycles between ground states $\mathrm{F}=2, \mathrm{~m}=2$ and excited states $\mathrm{F}^{\prime}=3, \mathrm{~m}^{\prime}=3$. The following figure represents the $D_{2}$ hyperfine structure of the sodium atom and the two-level cycle [11].

In Figure $1, D_{2}$ denotes the energy levels between $3 P_{3 / 2}$ and $3 S_{1 / 2}$ of sodium atoms, $D_{2 a}$ denotes the level transitions excited by the light with wavelength $589.159 \mathrm{~nm}, D_{2 b}$ 
denotes the level transitions excited by the light with wavelength $589.157 \mathrm{~nm}, \sigma^{+}$denotes the right-handed circularly polarized light, and the solid double arrows denote the twolevel cycle between $3 P_{3 / 2}(3,3)$ and $3 S_{1 / 2}(2,2)$. The left and second column data denote the frequency intervals between energy levels. The two-level cycle obtains a stable state in a microsecond order time for the continuous wave laser [12]. The probability of an excited sodium atom is written by [4]

$$
p_{2}=\frac{I / 2 I_{s a t}}{1+16\left[\pi \tau\left(v_{L}-v_{p}-v_{D}\right)\right]^{2}+I / I_{s a t}},
$$

where $v_{L}$ is the center frequency of laser, $v_{p}$ is the transition frequency of the two energylevel atoms, $I$ denotes laser intensity, $I_{\text {sat }}$ represents the saturated intensity, $I_{\text {sat }}=\pi h v /\left(3 \lambda^{2} \tau\right)$, $\tau$ is the lifetime of sodium atoms in the excited states, and $v$ is the radiative frequency of a photon.

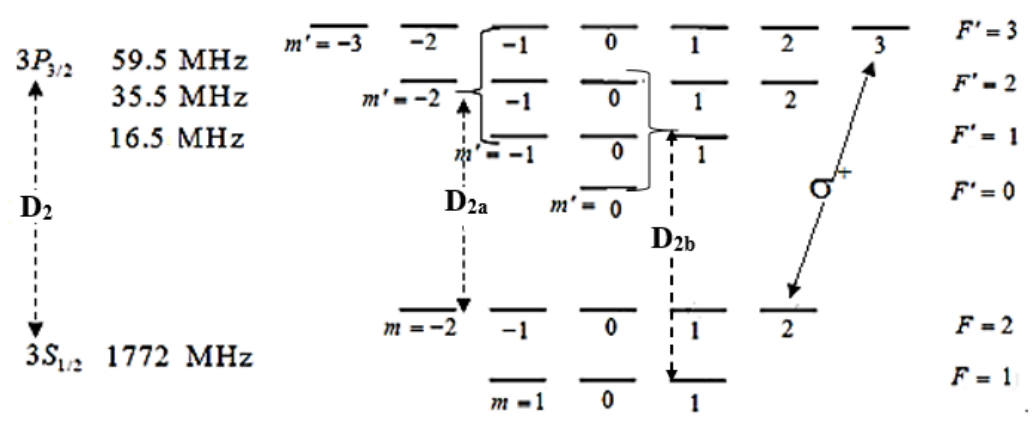

Figure 1. The $D_{2}$ hyperfine structure of sodium atom and the two-level cycle.

At the outset, the distribution of sodium atoms in the mesosphere satisfies the Maxwell velocity distribution law. The normalized distribution of sodium atoms is given by [13]

$$
N_{v_{D}}=\frac{(4 \ln 2 / \pi)^{1 / 2}}{\delta v_{D}} e^{-4 \ln 2 v_{D}^{2} /\left(\delta v_{D}\right)^{2}},
$$

where $N_{v_{D}}$ is the percent of sodium atoms corresponding to a Doppler shift, $\delta v_{D}$ denotes the linewidth of sodium atom distributions in the mesosphere, and $\int_{-\infty}^{+\infty} N_{v_{D}} d v_{D}=1$. If $\Delta v_{D}$ is regarded as a velocity interval, then the percent of sodium atoms in the small enough velocity interval is

$$
N_{v_{\mathrm{D}}}\left(\Delta v_{D}\right)=\frac{(4 \ln 2 / \pi)^{1 / 2}}{\delta v_{D}} e^{-4 \ln 2 v_{D}^{2} /\left(\delta v_{D}\right)^{2}} \Delta v_{D},
$$

For a single-mode laser, the intensity distribution with the Doppler shift, $v_{D}$, is written as [13]

$$
I_{0}\left(v_{D}\right)=I \frac{(4 \ln 2 / \pi)^{1 / 2}}{\delta v_{D}^{L}} e^{-4 \ln 2 v_{D}^{2} /\left(\delta v_{D}^{L}\right)^{2}},
$$

where $\delta v_{D}^{L}$ is the laser linewidth, and $I$ is the total intensity in a certain area illuminated by laser. The center of the laser intensity is coincident with that of the sodium atomic distributions. Thus, the peak probability of the excited sodium atoms can be obtained when the laser and sodium atoms satisfy the resonant conditions. The probability of excited sodium atoms at every $v_{D}$ is

$$
p_{2}\left(v_{D}\right)=\frac{I\left(v_{D}\right) / 2 I_{s a t}}{1+I\left(v_{D}\right) / I_{s a t}}
$$


where those sodium atoms away from the resonant conditions are taken into account. These atoms can be excited by near resonance. For a fixed value $I\left(v_{D}\right)$, the excitation probability of sodium atoms including near resonance is the following form [4]:

$$
p_{2}^{\prime}=\frac{I\left(v_{D}\right) / 2 I_{s a t}}{1+16\left[\pi \tau\left(v_{D}^{\prime}-v_{D}\right)\right]^{2}+I\left(v_{D}\right) / I_{\text {sat }}},
$$

where $v_{D}^{\prime}$ is the Doppler frequency shift relative to $v_{D}$.

To explicitly indicate the excited effectiveness of the ground sodium atoms, the concept of the average spontaneous emission rate is introduced. At the same time, the stimulated emission is ignored. For the CW laser, without regard to the geomagnetic field, the average spontaneous emission rate is

$$
\bar{R}=\frac{1}{\Delta T} \sum_{n} \iint_{-\infty}^{+\infty} \overline{5}\left(N_{v_{D}}^{\prime}\right)_{n} \cdot p_{2}^{\prime} d v_{D}^{\prime} d v_{D}
$$

where $\Delta T$ is the cycle time of sodium atomic collisions, $n$ denotes the times of excited sodium atomic upward transitions, $N_{v_{D}}^{\prime}$ denotes the sum of sodium atoms of decay from the excited states and the remainder in the ground states after the partial sodium atoms are excited every time, and 5/8 refers to the proportion of sodium atoms in the ground states corresponding to $\mathrm{m}=0, \pm 1, \pm 2$ [14]. Sodium atomic collisions, including velocity exchange, spin damping, and beam exchange, make numerous excited sodium atoms return the $\mathrm{F}=2$ ground states. Milonni [15] has estimated the velocity exchange time to be $100 \mu \mathrm{s}$. This implies that the motional states of all sodium atoms will make a return after $100 \mu$ s. However, Holzlöhner [2] has calculated the time to be $35 \mu \mathrm{s}$. In this article, $35 \mu \mathrm{s}$ is regarded as the cycle time.

In adaptive optics, enough return photons from the laser guide star are important for the wave-front detection [16]. For the continuous wave laser, the return photons in the unit area and the unit time on the telescope plane are written by [17]

$$
F_{\phi}=T_{0}^{\sec (\zeta)} \beta^{\prime} C_{N a} \bar{R} f_{m} \int_{s} d s /\left[4 \pi L^{2} \sec (\zeta)\right],
$$

where $T_{0}$ is the atmospheric transmissivity, $\beta^{\prime}$ is the backscattering coefficient of excited sodium atoms, $C_{\mathrm{Na}}$ is the column density of sodium atoms in the mesosphere, $L$ is the vertical distance from the telescope plane to the center of the mesospheric sodium layer, $\zeta$ is the zenith between the laser beam and the vertical direction, $s$ is the area illuminated by the laser, and $f_{m}$ is the scale factor of depolarization since the geomagnetic field cuts down on the number of sodium atoms in the $\mathrm{F}=2$ and $\mathrm{m}=2$ ground states [18]. Values of $f_{m}$ depend on the angles between the circular-polarized laser beam and the direction of the geomagnetic field and the period of Larmor precession. According to an experimental study [19], this factor can be reduced to $f_{m}=1-0.6552 B / B_{0} \sin \theta$, where $B$ and $B_{0}$ $\left(B_{0}=0.51 \mathrm{Gs}\right)$ are the magnitude of the geomagnetic field, and $\theta$ is the angle between the directions of the laser beam and the geomagnetic field vector.

According to Equations (7) and (8), $\bar{R}$ relates to laser intensity. Since laser propagation in the atmosphere is easily affected by atmospheric turbulence, laser intensity distributions present random states in the mesosphere. Laser field propagation accords to the following parabolic Equation [20]:

$$
\frac{\partial E}{\partial z}=\frac{i}{2 k_{1}} \nabla_{\perp}^{2} E+i k_{1} n_{1} E
$$

where $k_{1}$ stands for the wave number, $z$ is the path of laser propagation, $E$ is the amplitude of the light field, and $n_{1}$ denotes the fluctuation of the atmospheric refractive index around 1. By solving Equation (10), the light field at $z$ is achieved. Then, the laser intensity distributions are calculated. 
In addition to the return photons, the spot sizes of the sodium laser guide star are required to be small for the wave-front detection. The effective radius of spot size is exploited to characterize the energy focusability of the sodium laser guide star at the mesospheric sodium layer. This concept is defined as [21]

$$
R_{e f f}=\sqrt{2}\left[\iint r^{2} I_{b}(x, y) d x d y / \iint I_{b}(x, y) d x d y\right]^{1 / 2},
$$

where $I_{b}(x, y)$ is the fluorescent intensity of the sodium laser guide star at the sodium layer, observed from the orthogonal direction with two-dimensional coordinates $(x, y)$, and $r$ is the distance from $I_{b}(x, y)$ to the centroid of the light spot. $I_{b}(x, y)$ is calculated by the following expression:

$$
I_{b}(x, y)=T_{0}^{\sec (\zeta)} \beta^{\prime} C_{N a} \bar{R} f_{m} \Delta s \times h v,
$$

where $T_{0}^{\sec (\zeta)} \beta^{\prime} C_{N a} \bar{R} f_{m}$ denotes the backscattering photons of the sodium laser guide star in unit time, $\Delta \mathrm{s}$ is the very small area of radiative fluorescence which meets $\Delta s=\Delta x \Delta y$, and $h v$ stands for photon energy in $J$.

Based on the above analysis, we conclude that the recoil effects cause the red shifts of sodium atoms. Thus, a mass of sodium atoms miss excitation so that the spontaneous emission rate reduces when recoil occurs. In order to mitigate these effects, we propose that the laser linewidth should be broadened to weaken these recoil effects.

\section{Methods and Parameters}

\subsection{Numerical Simulation Methods}

To explore the linewidth broadening mitigating recoil effects of sodium laser guide star, numerical simulations are carried out. A fundamental assumption is that the two-energy level cycle of sodium atoms is able to be very well maintained due to enough re-pumping. Since the re-pumping power is about $10 \%$, even less than $10 \%$, in the total laser power [22], this power is ignored in the numerical simulations. The average spontaneous emission rates and return photons with respect to this power are attributed to the total values of the cycles between ground states $\mathrm{F}=2, \mathrm{~m}=2$ and excited states $\mathrm{F}^{\prime}=3, \mathrm{~m}^{\prime}=3$.

According to the theoretical models, Equations (3)-(10) are discretized. A numerically simulated method is employed to solve Equation (8). Its discrete formation is written as

$$
\bar{R}=\frac{1}{n \tau^{\prime}} \sum_{n} \sum_{i} \frac{5}{8}\left[N_{v_{D}}^{\prime}(i)\right]_{n} \cdot p_{2}^{\prime}(i) \Delta v_{D}^{\prime} \Delta v_{D},
$$

where $n \tau^{\prime}=\Delta T, \tau^{\prime}=2 \tau, \tau^{\prime}$ represents the time of decay and once again the excitation of a sodium atom, $i$ is defined as the number of velocity groups, $N_{v_{D}}^{\prime}(i)$ denotes the number of sodium atoms in the $i$-th velocity group, and $p_{2}^{\prime}(i)$ denotes the excitation probability of sodium atoms in Equation (7).

For the purpose of obtaining enough return photons, from Equations (7) and (8), $\bar{R}$ is required to be maximum under the same other parameters. We set 200001 velocity groups with the adjacent interval $\Delta v_{D}=1.0 \times 10^{4} \mathrm{~Hz}$. The range of Doppler shifts is taken from $-1.0 \mathrm{GHz}$ to $1.0 \mathrm{GHz}$.

To solve Equation (10), multi-phase screen method [23] is employed. Moreover, the atmospheric turbulence model of Greenwood [24] and power spectrum of Kolmogorov [25] are used in simulations of laser atmospheric propagation. Laser intensity distributions are discretized as $512 \times 512$ grids. Laser intensity is thought as concentrating on a plane through the whole sodium layer. Then, the return photons are calculated according to Equation (9). Similarly, Equation (11) is discretized as the following form [21]: 


$$
R_{e f f}=\sqrt{2}\left[\sum_{m, n} r_{m, n}^{2} I_{b}(m, n) \Delta s / \sum_{m, n} I_{b}(m, n) \Delta s\right]^{1 / 2}
$$

where $I_{b}(m, n)$ is intensity of sodium laser guide star in the $m$-th row and $n$-th column, and $m$ and $n$ are, respectively, the row and column ordinals of $512 \times 512$ grids. Due to the effects of atmospheric turbulence, the distribution of laser intensity is randomized in the mesospheric sodium layer. To simulate laser intensity, the multi-phase screen method is used to solve Equation (10) [23]. The power spectrum of Kolmogorov turbulence is taken into account, and its expression is [24]

$$
\Phi(k)=0.033 r_{0}^{-5 / 3} k^{-11 / 3}
$$

where $r_{0}$ is atmospheric coherent length, $k$ is spatial frequency, $r_{0}=0.185\left[\frac{\lambda}{\int_{0}^{h^{\prime}} C_{n}^{2}(\xi) \mathrm{d} \xi}\right]^{3 / 5}$, $C_{\mathrm{n}}^{2}$ is refractive index structure constant for atmosphere, and $h^{\prime}$ is the atmospheric vertical height from the ground in $\mathrm{m}$. The atmospheric turbulence model of Greenwood is [25]

$$
C_{\mathrm{n}}^{2}\left(h^{\prime}\right)=\left[2.2 \times 10^{-13}\left(h^{\prime}+10\right)^{-13}+4.3 \times 10^{-17}\right] e^{-h^{\prime} / 4000} .
$$

On the thin layer perpendicular to the laser transmission direction, the power spectrum of atmospheric phase is written as [26]

$$
\Phi_{n}(k)=2 \pi(2 \pi / \lambda)^{2} 0.033 k^{-11 / 3} \int_{z}^{z+\Delta z} C_{\mathrm{n}}^{2}(\xi) \mathrm{d} \xi .
$$

Then, Equation (17) is filtered by a complex Gaussian random matrix $a^{\prime}(m, n)$ and through the inverse Fourier transform to the discretized phase screen as follows [27]:

$$
\phi^{\prime}(m, n)=\sum_{m^{\prime}=1}^{N_{x}} \sum_{n^{\prime}=1}^{N_{y}} a^{\prime}(m, n)\left(\frac{0.479}{L_{x} L_{y}} r_{0}^{-5 / 6} k^{-11 / 6}\right) \cdot \exp \left[j 2 \pi\left(\frac{m m^{\prime}}{N_{x}}+\frac{n n^{\prime}}{N_{y}}\right)\right],
$$

where $L_{x}$ and $L_{y}$ are side lengths, and $N_{x}$ and $N_{y}$ are the number of grids. Furthermore, the third harmonic method is used to compensate for the low frequency inadequacy. Finally, the total phase $S(r, z)$, including the low and high frequency components, modulates the light field. Thus, the solution of Equation (10) is expressed as [28]

$$
\boldsymbol{E}(\boldsymbol{r}, \underline{z+\Delta z})=\exp \left[\frac{i}{2 k} \int_{z}^{z+\Delta z} \nabla_{\perp}^{2} \mathrm{~d} \xi\right] \cdot \exp [\mathrm{iS}(\boldsymbol{r}, z)] \boldsymbol{E}(\boldsymbol{r}, z),
$$

where $\exp \left[\frac{i}{2 k} \int_{z}^{z+\Delta z} \nabla_{\perp}^{2} \mathrm{~d} \xi\right]$ is caused by vacuum diffraction.

\subsection{Simulation Parameters}

This simulation study involves laser characteristics, atmospheric properties, and sodium layer features. All relevant parameters are listed in Table 1 [2].

When $\theta=30^{\circ}$ and $B=0.228 \mathrm{Gs}$, the scale factor of depolarization $f_{m}=0.8466$. Specifically, a laser with TEM00 mode is launched at collimation. 
Table 1. Numerical simulation parameters.

\begin{tabular}{lcl}
\hline Variable Names & Symbols & Values \\
\hline Laser parameters & & \\
Center wavelength of laser & $\lambda$ & $589.159 \mathrm{~nm}$ \\
Linewidth of continuous wave laser & $\delta v_{D}^{L}$ & $0-1.0 \mathrm{GHz}$ \\
Laser polarization & $\sigma^{+}$ & circular \\
Laser beam quality factor & $\beta$ & 1.1 \\
Diameter of laser launch & $D$ & $40 \mathrm{~cm}$ \\
Zenith of laser launch & $\zeta$ & $30^{\circ}$ \\
Angle between directions of laser beam & & \\
and geomagnetic field vector & $\theta$ & $30^{\circ}$ \\
Sodium parameters & & \\
Linewidth of sodium atomic distributions at sodium layer & $\delta v_{D}$ & $1.0 \mathrm{GHz}$ \\
Life time of excited sodium atoms & $\tau$ & $16 \mathrm{~ns}$ \\
Backscattering coefficient of excited sodium atoms & $\beta^{\prime}$ & 1.5 \\
Column density of sodium layer & $C_{\mathrm{Na}}$ & $4 \times 10^{13} \mathrm{~cm}^{-2}$ \\
Cycle time of sodium atomic collisions & $\Delta T$ & $35 \mu \mathrm{s}$ \\
Altitude of sodium layer centroid & $L$ & $92 \mathrm{~km}$ \\
Atmospheric, magnetic field parameters & & \\
Atmospheric transmissivity & $T_{0}$ & 0.8 \\
Mesospheric magnetic field & $B$ & $0.228 \mathrm{Gs}$ \\
\hline
\end{tabular}

\section{Results and Analysis}

\subsection{Recoil and Linewidth Broadening}

The continuous wave laser is single-mode with a 0 or $2.0 \mathrm{MHz}$ linewidth. For the 2.0 MHz linewidth laser, its intensity distribution is expressed as Equation (5). The total intensity of the laser is taken as $I=150 \mathrm{~W} / \mathrm{m}^{2}$. It is assumed that sodium atoms are excited every 32 ns due to the cycle time of excited states. The tens of nanoseconds in the ascending stage are ignored before steady states. For the $0 \mathrm{MHz}$ laser, the normalized distributions of sodium atoms after recoil are simulated at $t=10 \mu \mathrm{s}, 20 \mu \mathrm{s}$, and $35 \mu \mathrm{s}$ as in Figure 2. In order to study the effects of linewidth broadening on the mitigation of recoil, the linewidth of the continuous wave laser is taken to be $2.0 \mathrm{MHz}$ in Equation (5). After $t=10 \mu \mathrm{s}, 20 \mu \mathrm{s}$, and $35 \mu \mathrm{s}$, the normalized distributions of the sodium atoms are presented in Figure 3.

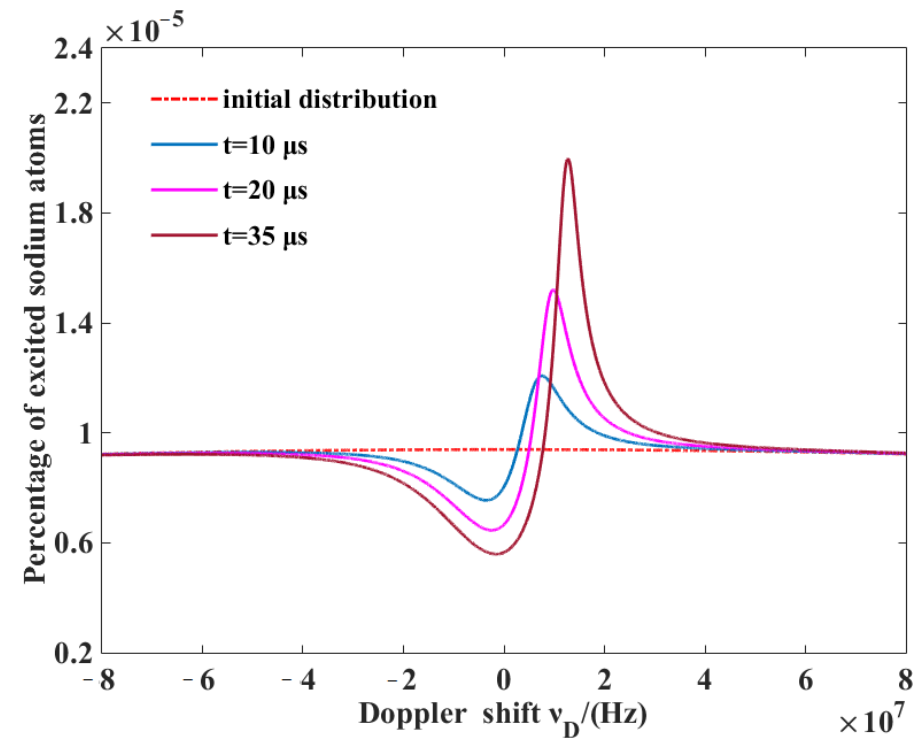

Figure 2. Normalized distributions of sodium atoms with recoil at $t=10 \mu \mathrm{s}, 20 \mu \mathrm{s}$, and $35 \mu \mathrm{s}$ for $0 \mathrm{MHz}$ linewidth. 


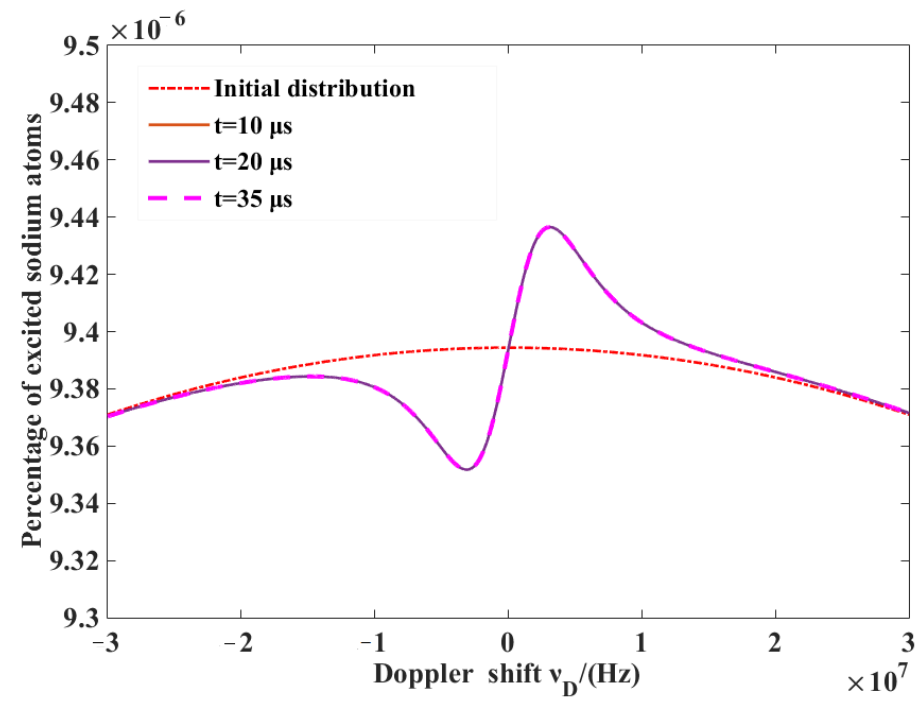

Figure 3. Normalized distributions of sodium atoms with linewidth broadening at $t=10 \mu \mathrm{s}, 20 \mu \mathrm{s}$, and $35 \mu \mathrm{s}$.

From Figure 2, one can see that recoil results in the accumulation of sodium atoms at higher and higher Doppler shifts as time goes on. Compared with Figure 2, after linewidth broadening is employed, the peaks of recoil greatly drop in Figure 4, and the corresponding three sodium atomic distributions are coincident. In addition to this, the laser intensity also influences recoil, as is shown in Figure 4 . With the same linewidth broadening method as the above, after $\mathrm{t}=35 \mu \mathrm{s}$ for $I=50 \mathrm{~W} / \mathrm{m}^{2}, 100 \mathrm{~W} / \mathrm{m}^{2}$, and $150 \mathrm{~W} / \mathrm{m}^{2}$, the situations of mitigated recoil are shown in Figure 5.

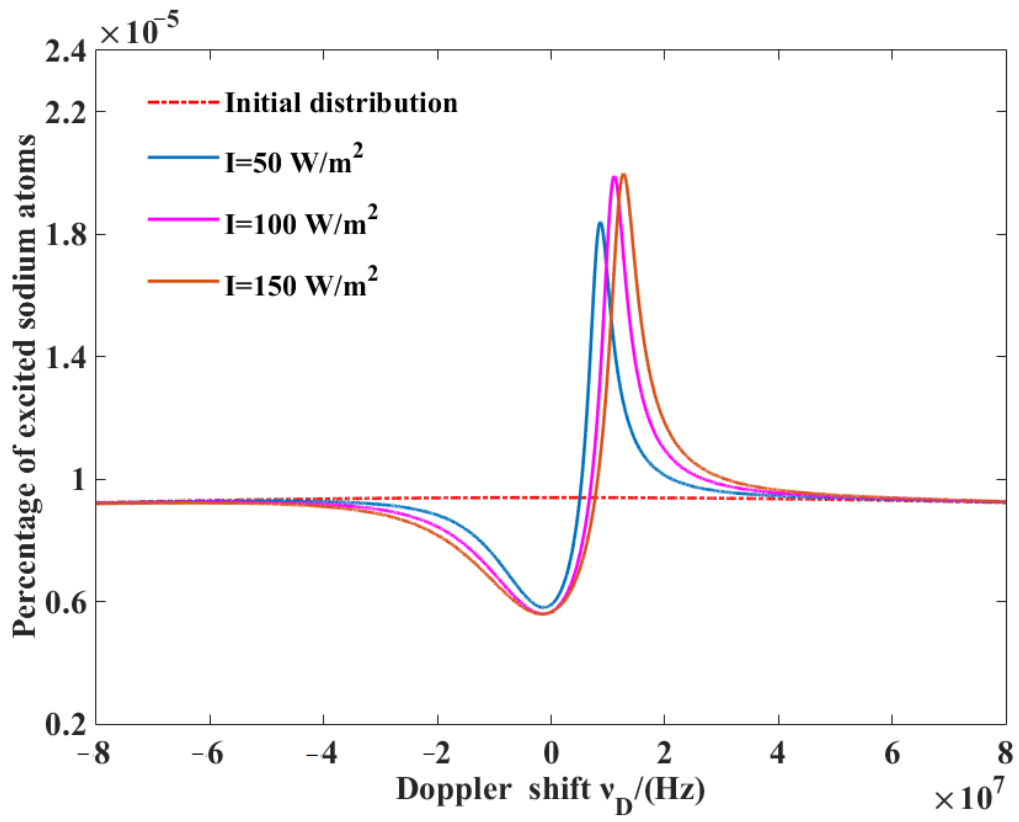

Figure 4. Normalized distributions of sodium atoms with recoil for $I=50 \mathrm{~W} / \mathrm{m}^{2}, 100 \mathrm{~W} / \mathrm{m}^{2}$, and $150 \mathrm{~W} / \mathrm{m}^{2}$ for $0 \mathrm{MHz}$ linewidth. 


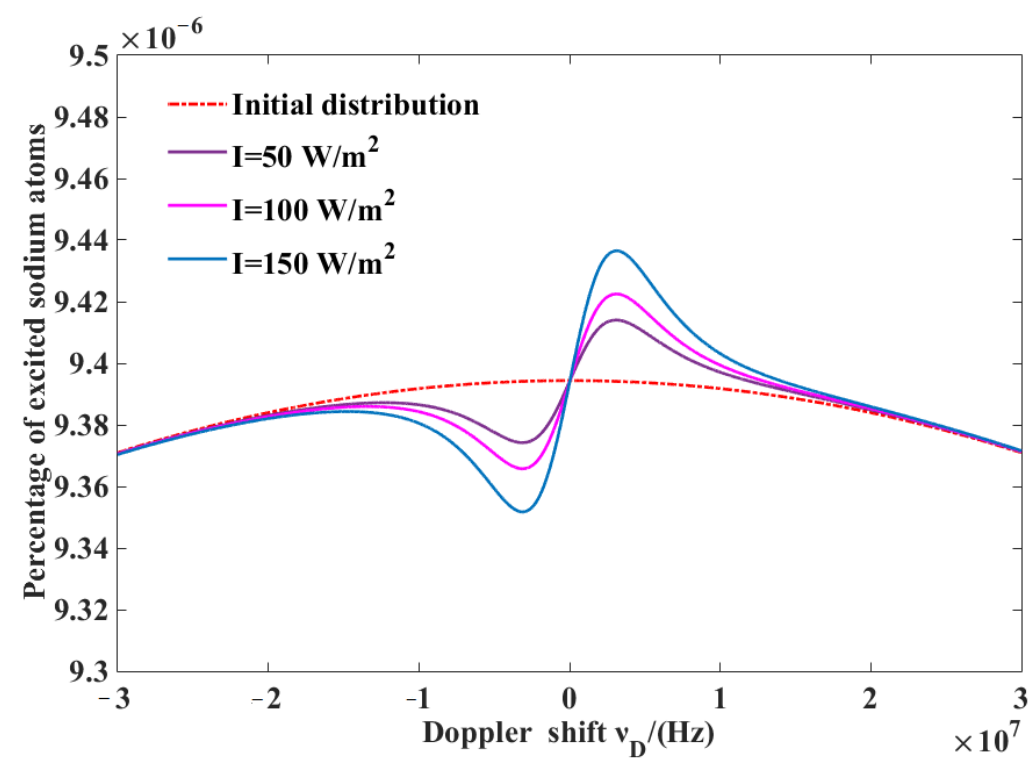

Figure 5. Normalized distributions of sodium atoms with linewidth broadening for $I=50 \mathrm{~W} / \mathrm{m}^{2}$, $100 \mathrm{~W} / \mathrm{m}^{2}$, and $150 \mathrm{~W} / \mathrm{m}^{2}$ for $0 \mathrm{MHz}$ linewidth.

Figure 4 shows that high intensity causes more drastic recoil and aggravates the adverse situations. Simultaneously, the higher intensity makes sodium atoms drift to the higher Doppler frequency shifts. Figure 5 reveals that the linewidth broadening method can effectively alleviate the recoil effects for different laser intensities.

\subsection{Choice of Optimal Laser Linewidth}

In practice, if the recoil effects have to be dropped, and the laser is required to modulate the intensity distribution in Equation (5). The linewidth broadening of the laser intensity distribution aims at achieving the maximal excitation probability of mesospheric sodium atoms. The maximal average spontaneous emission rate is necessary. Therefore, we simulate the average spontaneous emission rates by the linewidth broadening from 0 to 1.0 GHz. In light of Equations (2)-(9), the average spontaneous emission rates with the intensity from 0 to $1500 \mathrm{~W} / \mathrm{m}^{2}$ are simulated in Figures 6 and 7 .

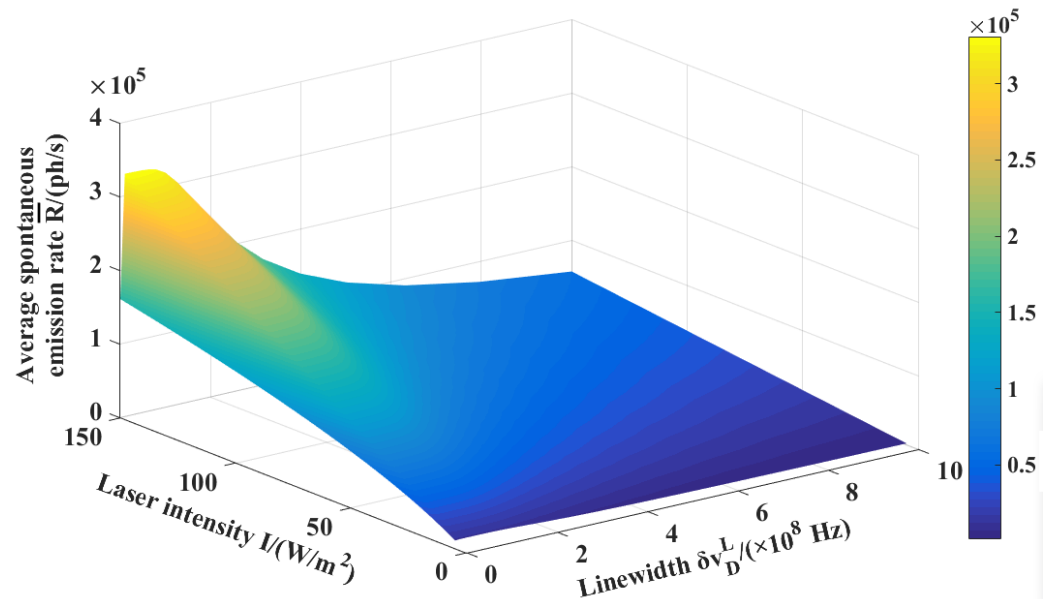

Figure 6. Average spontaneous emission rates vs. linewidth and intensity from 5 to $150 \mathrm{~W} / \mathrm{m}^{2}$. 


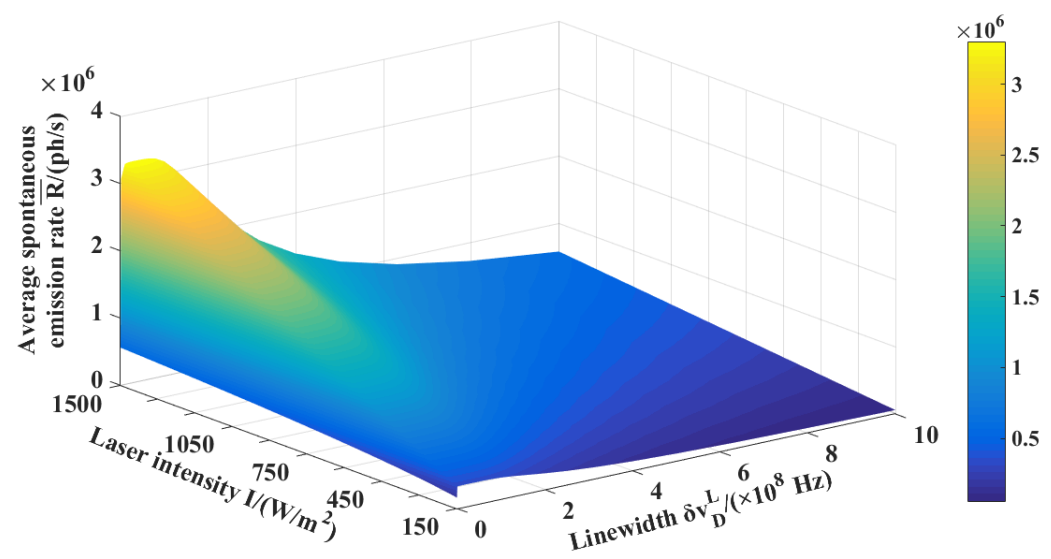

Figure 7. Average spontaneous emission rates vs. linewidth and intensity from 150 to $1500 \mathrm{~W} / \mathrm{m}^{2}$.

Figures 6 and 7 show that the peak values of average spontaneous emission rates change with the laser linewidth and intensity. The high intensity enhances the peak values of average spontaneous emission rates.

When the laser is broadened to a larger linewidth, the average spontaneous emission rates instead drop. In the case of lower intensity, the laser linewidth broadening finitely gains the average spontaneous emission rates in the range of $1-100 \mathrm{MHz}$. However, it is not that the wider linewidth can obtain the best effect, but that the average spontaneous emission rates have a maximum for the linewidth from $1 \mathrm{MHz}$ to $100 \mathrm{MHz}$. However, the average spontaneous emission rate at $\delta v_{D}^{L}=0 \mathrm{MHz}$ is lower than the peak values. In Figures 6 and 7 , the peak values of average spontaneous emission rates are the same in terms of linewidth.

We hope that the linewidth broadening of laser intensity distributions makes the average spontaneous emission rate maximal. Figures 8 and 9 simulate the average spontaneous emission rates for laser linewidth from 1 to $10^{3} \mathrm{MHz}$ and laser intensity from 5 to $1500 \mathrm{~W} / \mathrm{m}^{2}$.

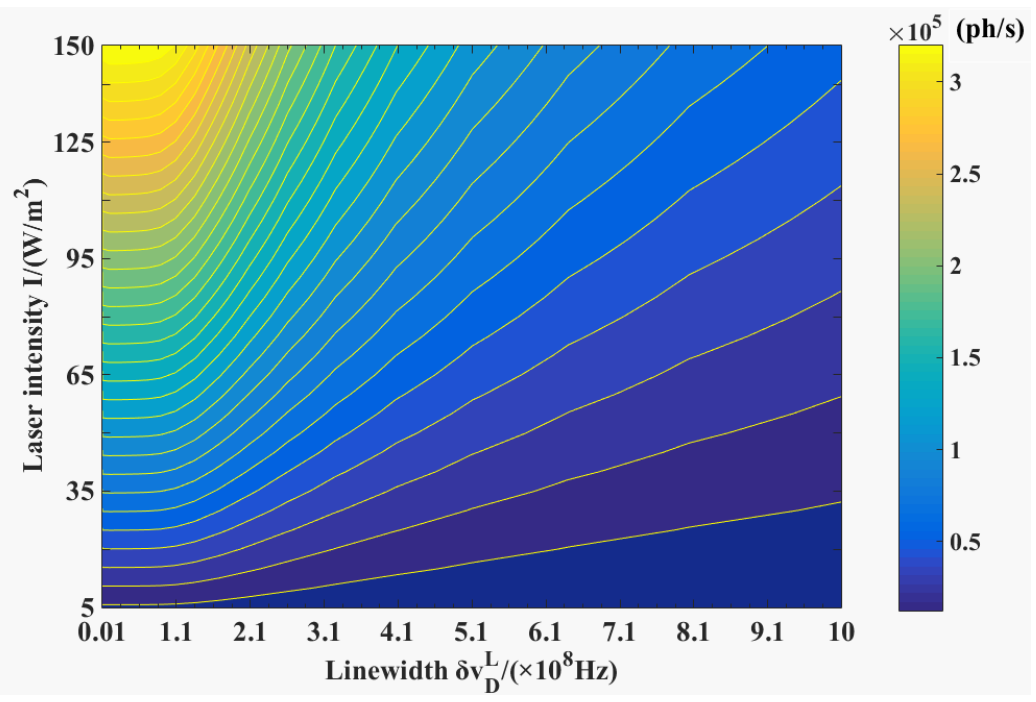

Figure 8. Average spontaneous emission rates for laser linewidth from 3 to $10^{3} \mathrm{MHz}$ and laser intensity $I=5-150 \mathrm{~W} / \mathrm{m}^{2}$. 


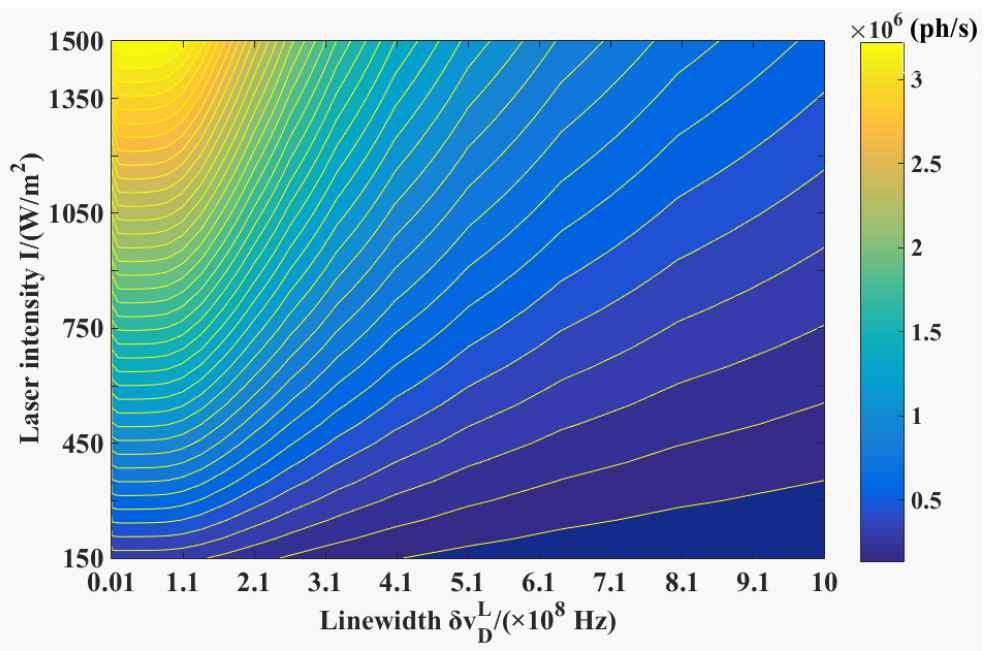

Figure 9. Average spontaneous emission rates for laser linewidth from 3 to $10^{3} \mathrm{MHz}$ and laser intensity $I=150-1500 \mathrm{~W} / \mathrm{m}^{2}$.

Figures 8 and 9 indicate that the peak values of average spontaneous emission rates are between $1 \mathrm{MHz}$ and $100 \mathrm{MHz}$ for an intensity from $5 \mathrm{~W} / \mathrm{m}^{2}$ to $1500 \mathrm{~W} / \mathrm{m}^{2}$. Therefore, the laser linewidth is taken as the value between $1 \mathrm{MHz}$ and $100 \mathrm{MHz}$. Figure 10 demonstrates the relation between laser linewidth at $\delta v_{D}^{L}=0,1,10,100 \mathrm{MHz}$ and average spontaneous emission rates.

By comparing average spontaneous emission rates for every linewidth at $\delta v_{D}^{L}=0,1$, $10,100 \mathrm{MHz}$, the average spontaneous emission rates are lowest at $\delta v_{D}^{L}=0 \mathrm{MHz}$ and approximately equal for linewidth at $\delta v_{D}^{L}=1,10,100 \mathrm{MHz}$. This implies more return photons for the laser linewidth at $\delta v_{D}^{L}=1,10,100 \mathrm{MHz}$. The laser linewidth at $\delta v_{D}^{L}=10 \mathrm{MHz}$ is chosen to calculate the return photons. According to Figure 10, the relations between laser intensity and average spontaneous emission rates are fitted by

$$
\begin{aligned}
& \text { for } \delta_{D}^{L}=0 \quad \mathrm{MHz}, \quad \bar{R}=\frac{1.6153 \times 10^{5} I}{1+0.0033 I}, \\
& \text { for } \delta_{D}^{L}=10 \quad \mathrm{MHz}, \quad \bar{R}=2.169 \times 10^{3} I .
\end{aligned}
$$

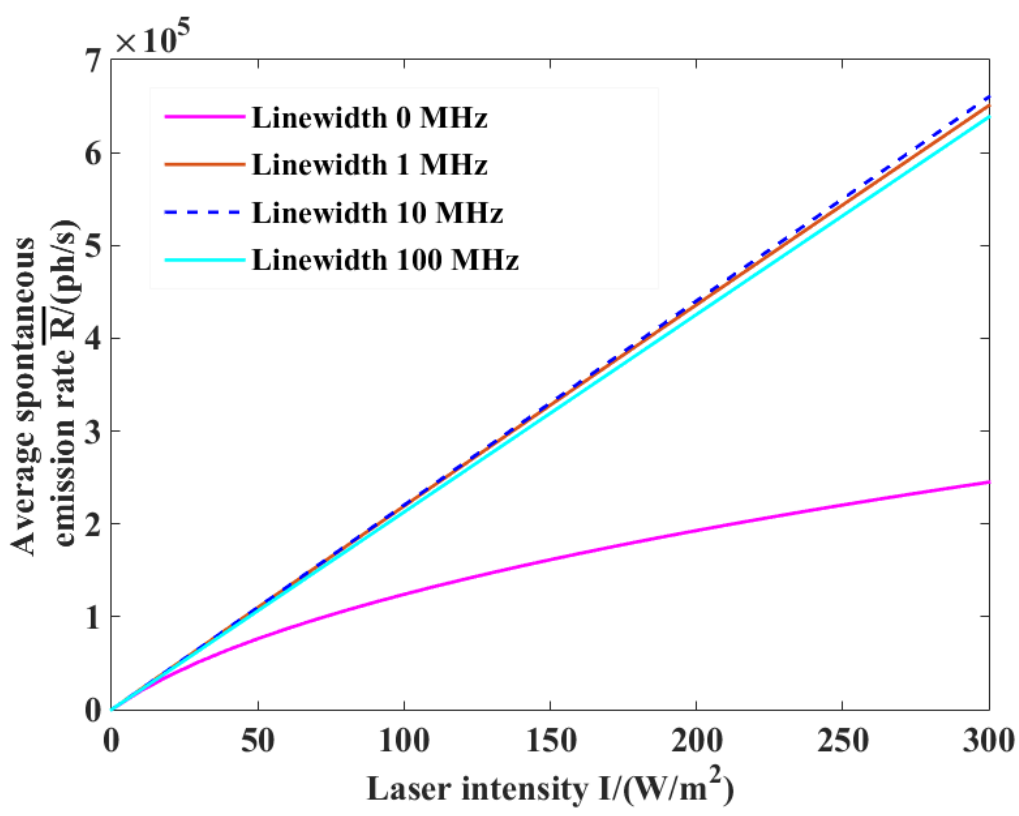

Figure 10. Average spontaneous emission rates vs. intensity at $\delta v_{D}^{L}=0,1,10,100 \mathrm{MHz}$. 


\section{Discussions}

\subsection{Effects of Linewidth Broadening on the Return Photons and Spot Sizes}

Generally, the higher laser power forms higher peak values of intensity in the mesospheric sodium layer. In accordance with the simulation method and parameters in Section 3, the return photons are calculated for a laser power from $10 \mathrm{~W}$ to $60 \mathrm{~W}$ at $\delta v_{D}^{L}=0$ and $10 \mathrm{MHz}$. Meanwhile, the spot sizes of the sodium laser guide star are computed. These data are listed in Table 2.

Table 2. Return photons and effective radii of spot sizes.

\begin{tabular}{|c|c|c|c|c|c|c|}
\hline Laser Power (W) & \multicolumn{2}{|c|}{10} & \multicolumn{2}{|c|}{20} & \multicolumn{2}{|c|}{30} \\
\hline $\begin{array}{l}\text { Laser linewidth } \\
\delta v_{D}^{L}(\mathrm{MHz}) \\
\text { Return photons }\end{array}$ & 10 & 0 & 10 & 0 & 10 & 0 \\
\hline$F_{\phi}\left(\times 10^{6} \mathrm{ph} / \mathrm{m}^{2} / \mathrm{s}\right)$ & 5.36 & 3.56 & 10.72 & 6.50 & 16.08 & 9.10 \\
\hline $\begin{array}{l}\text { Effective radius } \\
R_{\text {eff }} \quad(\mathrm{m})\end{array}$ & 0.426 & 0.426 & 0.418 & 0.418 & 0.422 & 0.422 \\
\hline Laser Power (W) & \multicolumn{2}{|c|}{40} & \multicolumn{2}{|c|}{50} & \multicolumn{2}{|c|}{60} \\
\hline $\begin{array}{l}\text { Laser linewidth } \\
\delta v_{D}^{L}(\mathrm{MHz}) \\
\text { Return photons }\end{array}$ & 10 & 0 & 10 & 0 & 10 & 0 \\
\hline$\underline{F_{\phi}}\left(\times 10^{6} \mathrm{ph} / \mathrm{m}^{2} / \mathrm{s}\right)$ & 21.43 & 11.26 & 26.795 & 13.05 & 32.15 & 15.29 \\
\hline $\begin{array}{l}\overline{\text { Effective radius }} \\
R_{e f f} \quad(\mathrm{~m})\end{array}$ & 0.413 & 0.413 & 0.406 & 0.406 & 0.413 & 0.413 \\
\hline
\end{tabular}

Based on the above data, we can summarize three results. First, linewidth broadening is able to achieve the most return photons. We find that the laser linewidth at $\delta v_{D}^{L}=10 \mathrm{MHz}$ produces more return photons than that at $=0 \mathrm{MHz}$. Compared with that at $\delta v_{D}^{L}=0$, the return photons at $\delta v_{D}^{L}=10 \mathrm{MHz}$ increase $50.4 \%$. Second, increments of the return photons increase with laser power. For laser power from $10 \mathrm{~W}$ to $60 \mathrm{~W}$, calculations show that the increment of return photons goes up to $110 \%$ from $50.4 \%$. Thirdly, in general, the effective radii at $\delta v_{D}^{L}=10 \mathrm{MHz}$ are equal to those at $\delta v_{D}^{L}=0 \mathrm{MHz}$.

Therefore, the linewidth broadening method is useful to improve the signal-to-noise ratio of wave-front detection in adaptive optics.

It is well known that the strength of atmospheric turbulence can affect the intensity distribution of the laser. For the atmospheric turbulence model of Greenwood, the whole atmospheric coherent length is $15.6 \mathrm{~cm}$ (perpendicular to ground upward for wavelength $500 \mathrm{~nm}$ ). When the whole atmospheric coherent length is $21.6 \mathrm{~cm}$, such as the atmospheric turbulence model of Mod-HV [29], the intensity distribution of the laser will trend to the higher values in space. However, because linewidth broadening leads to the decrease of peak intensity in the spectrum, effects of recoil will be weakened .

\subsection{Considerations of Linewidth Broadening Method}

The two energy-level cycles of sodium atoms are influenced by several factors. Among them, depolarizations of the geomagnetic field enable a number of sodium atoms detuning to $\mathrm{F}=2 \mathrm{~m}=2 \leftrightarrow \mathrm{F}^{\prime}=3 \mathrm{~m}^{\prime}=3$ cycles. To maintain these two energy-level cycles, repumping ( $D_{2 b}$ in Figure 1 ) is used to excite the sodium atoms, trapping in $\mathrm{F}=1$ ground states [2]. The laser is generally modulated with a sideband by $1.713 \mathrm{GHz}$. This sideband linewidth should be broadened at $\delta v_{D}^{L}=1-100 \mathrm{MHz}$. Otherwise, the recoil will weaken the efficiency of re-pumping.

The single-frequency CW laser was once used to excite the sodium laser guide star [30]. This laser's power is $4-5 \mathrm{~W}$, but the efficiency is low because of the $0 \mathrm{MHz}$ linewidth. A modeless $\mathrm{CW}$ laser with a $3 \mathrm{GHz}$ linewidth is thought to be more efficient due to the wide 
spectrum, but its efficiency is not very high according to the above analysis [31]. The most famous CW laser is the 50-watt FASOR at the Starfire Optical Range [32]. On 30 May 2006, the experimental average value of the return photons was about $1.8 \times 10^{7} \mathrm{ph} / \mathrm{m}^{2} / \mathrm{s}$ for the 40 -watt FASOR, and the zenith of the laser launch was 0 degrees. Furthermore, the angle of the laser beam and geomagnetic field vector was about 28 degrees, similar to the 30 degrees in Table 1. Moreover, the column density of the sodium layer is nearly as much as $4 \times 10^{13} \mathrm{~cm}^{-2}$, and the atmospheric transmission is 0.91 . In particular, the laser linewidth is $1 \times 10^{4} \mathrm{~Hz}$ instead of $0 \mathrm{MHz}$. Thus, the average spontaneous emission rates are more than that of the $0 \mathrm{MHz}$ linewidth laser.Therefore, in Table 2, the calculated value of the return photons for the $0 \mathrm{MHz}$ linewidth laser with $40 \mathrm{~W}$ is basically in line with the actual situation. Figure 11 depicts the average spontaneous emission rates for the laser linewidth at $\delta v_{D}^{L}=0,0.01,1 \mathrm{MHz}$.

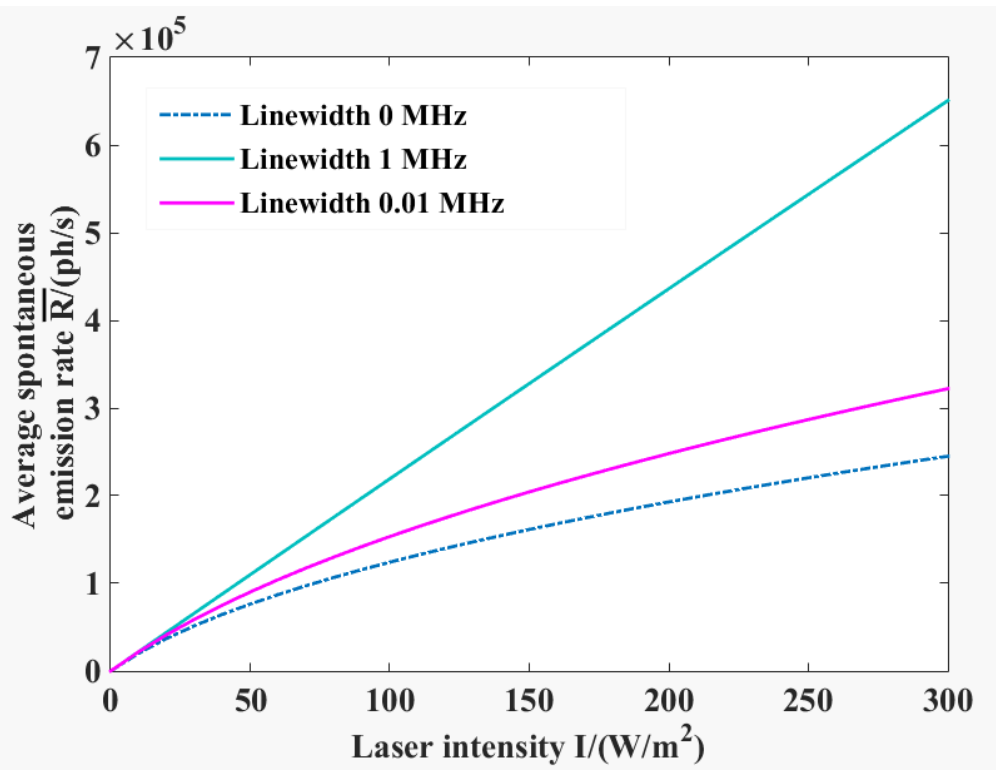

Figure 11. Average spontaneous emission rates vs. intensity at $\delta v_{D}^{L}=0,0.01,1 \mathrm{MHz}$.

From Figure 11, one can see that the average spontaneous emission rates at $\delta v_{D}^{L}=0.01 \mathrm{MHz}$ are the same as half at $\delta v_{D}^{L}=1 \mathrm{MHz}$ for a laser intensity more than $25 \mathrm{~W} / \mathrm{m}^{2}$. If the linewidth of the CW laser at the Starfire Optical Range is broadened to be $1 \mathrm{MHz}$, the return photons will increase about one times. At Gemini North (Mauna Kea), the CW laser, which is mono-mode, has a linewidth at $10 \mathrm{MHz}$, and has a zenith of 45 degrees, the return photons are simulated to be $2.22 \times 10^{6} \mathrm{ph} / \mathrm{m}^{2} / \mathrm{s}$ by an approximately $10 \mathrm{~W}$ laser [33].

The column density of the sodium layer is merely $2 \times 10^{13} \mathrm{~cm}^{-2}$. If this parameter is taken as $4 \times 10^{13} \mathrm{~cm}^{-2}$ in Table 1 , the calculated value is approximately $4.44 \times 10^{6} \mathrm{ph} / \mathrm{m}^{2} / \mathrm{s}$. This result is relatively close to $5.36 \times 10^{6} \mathrm{ph} / \mathrm{m}^{2} / \mathrm{s}$ in Table 2 .

\subsection{Effects of Linewidth Broadening on Recoil of the Multi-Mode Laser}

The multi-mode CW laser which is utilized to excite the sodium laser guide star has the advantage of a wide bandwidth. However, its efficiency is not seemingly as good as a single-mode laser [33].This laser has several centers of frequency. The expression of the laser intensity is [8]

$$
I\left(v_{D}\right)=I(0) \exp \left[-4 \ln 2\left(v_{\mathrm{D}} / \delta v_{b}\right)^{2}\right] \times \sum_{j=-k^{\prime}}^{+k^{\prime}} \exp \left[-4 \ln 2\left(v_{\mathrm{D}}-j v_{1}\right)^{2} / \delta v_{0}^{2}\right],
$$

where $j$ stands for the number of modes, $k^{\prime}$ is an integer, $v_{1}$ is an interval of adjacent modes, $v_{D}$ is the Doppler shift, $I\left(v_{D}\right)=I(0) \exp \left[-4 \ln 2\left(v_{D} / \delta v_{b}\right)^{2}\right]$ is the envelop curve, $I(0)$ is the peak power, and $\delta v_{b}$ is the linewidth of envelop curve. $\delta v_{0}$ is the mode linewidth. 
Taking $v_{1}=150 \mathrm{MHz}, k^{\prime}=1, \delta v_{0}=10^{4} \mathrm{~Hz}$, and $\delta v_{b}=1 \mathrm{GHz}$ results in a laser spectrum with three modes as in Figure 12.

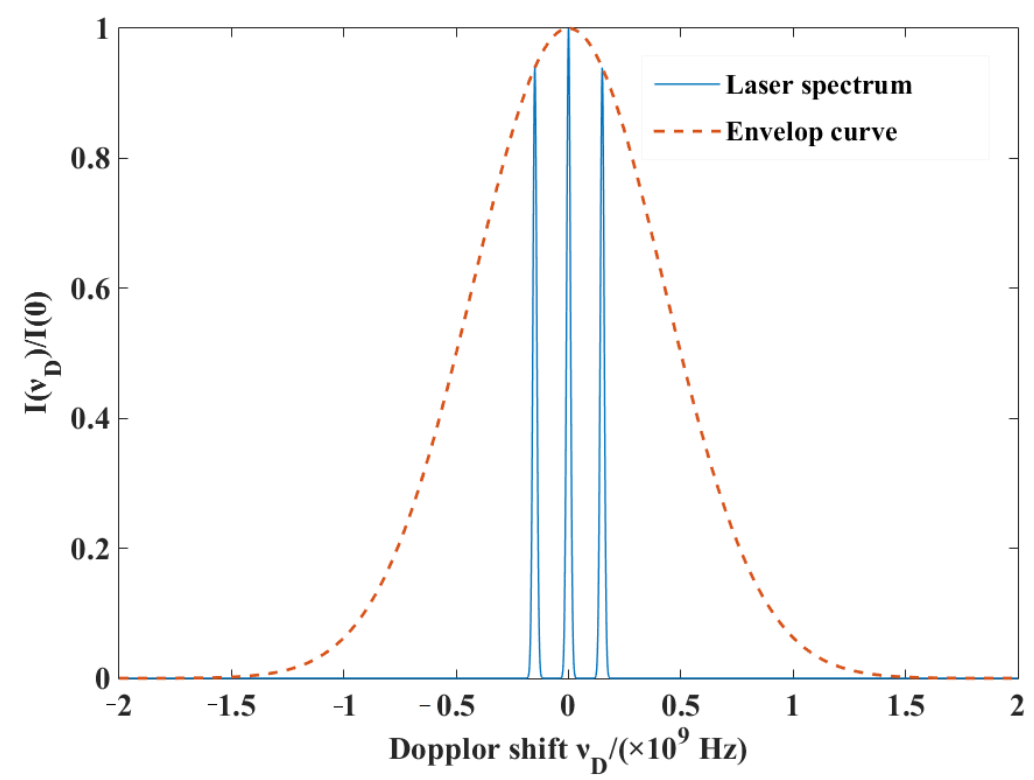

Figure 12. A laser spectrum with three modes at $\delta v_{0}=10^{4} \mathrm{~Hz}$. (The maximum value is normalized.)

When the laser mode linewidth is broadened to be $60 \mathrm{MHz}$, the laser spectrum with three modes is shown as Figure 13.

In light of the above simulated method, at $I=150 \mathrm{~W} / \mathrm{m}^{2}$, we simulate linewidth broadening influences on recoil in Figure 14. Results indicate that the linewidth broadening of three modes effectively weakens the recoil peaks of the distribution of sodium atoms.

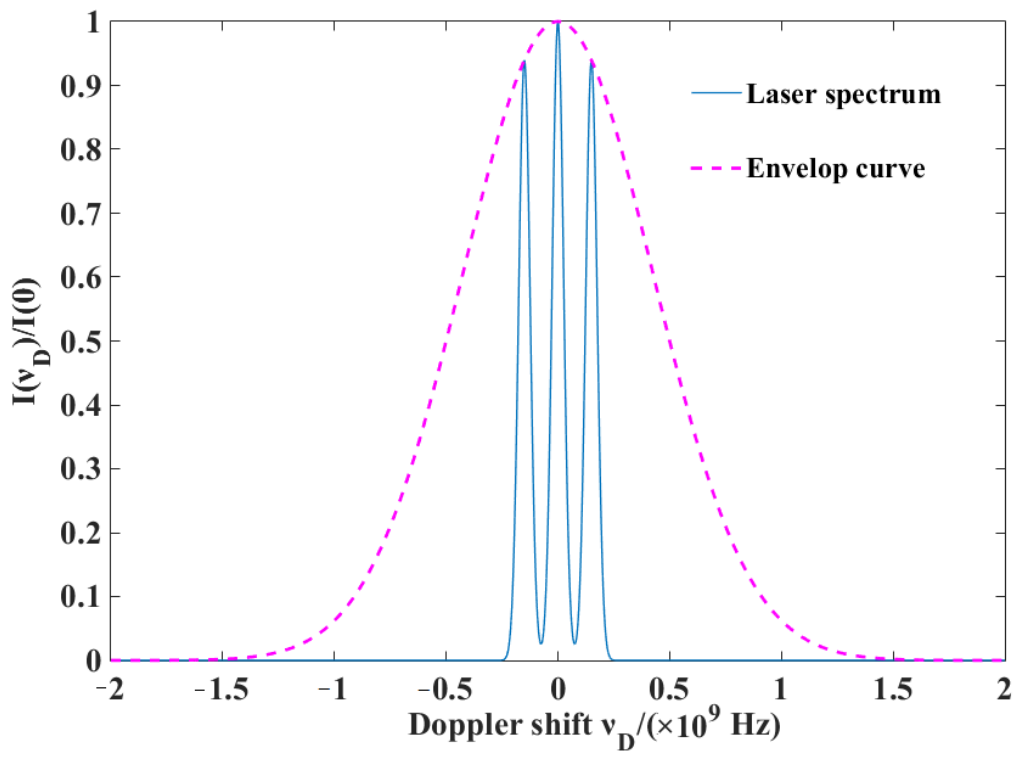

Figure 13. A laser spectrum with three modes at $\delta v_{0}=6 \times 10^{7} \mathrm{~Hz}$. (The maximum value is normalized.)

Figure 15 depicts the average spontaneous emission rates for the laser intensity from 0 to $200 \mathrm{~W} / \mathrm{m}^{2}$ at $\delta v_{0}=10^{4} \mathrm{~Hz}$ and $60 \mathrm{MHz}$ for the three-mode laser and at $\delta_{D}^{L}=0 \mathrm{MHz}$ for the mono-mode laser. 


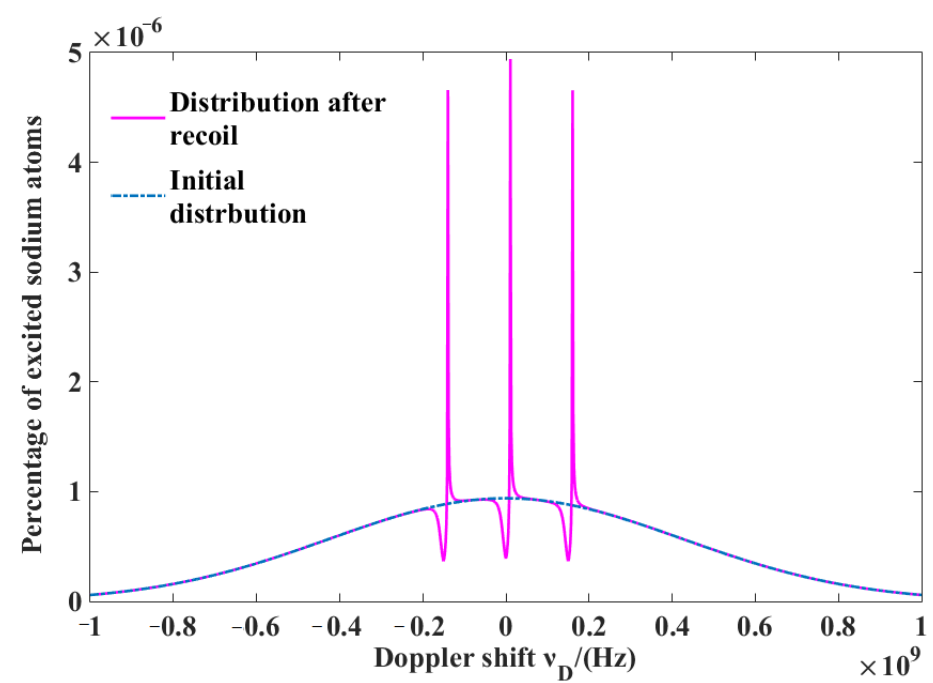

(a) Laser linewidth $10^{4} \mathrm{~Hz}$.

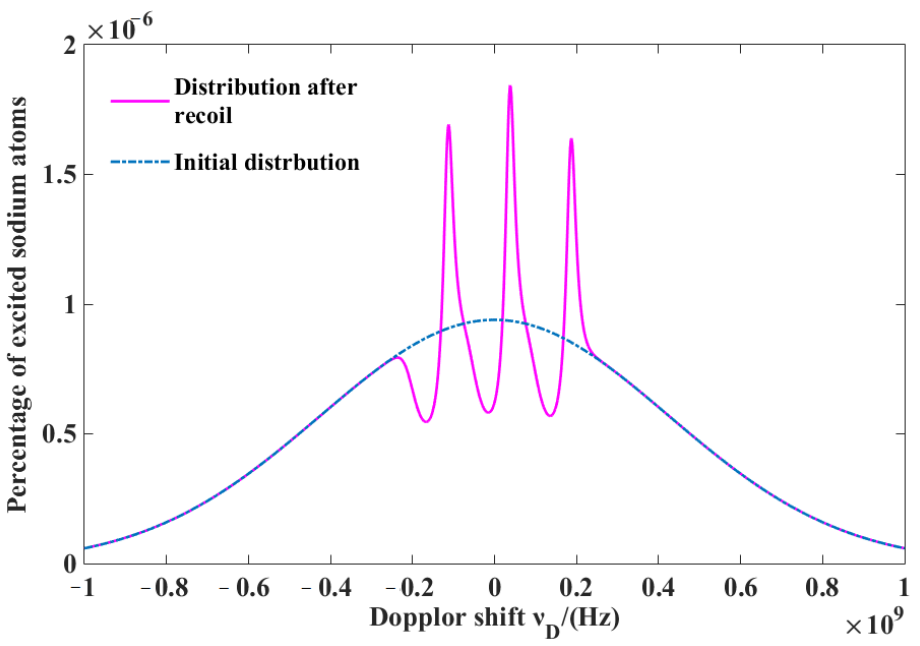

(b) Laser linewidth $60 \times 10^{6} \mathrm{~Hz}$.

Figure 14. Normalized distributions of sodium atoms at $I=150 \mathrm{~W} / \mathrm{m}^{2}$.

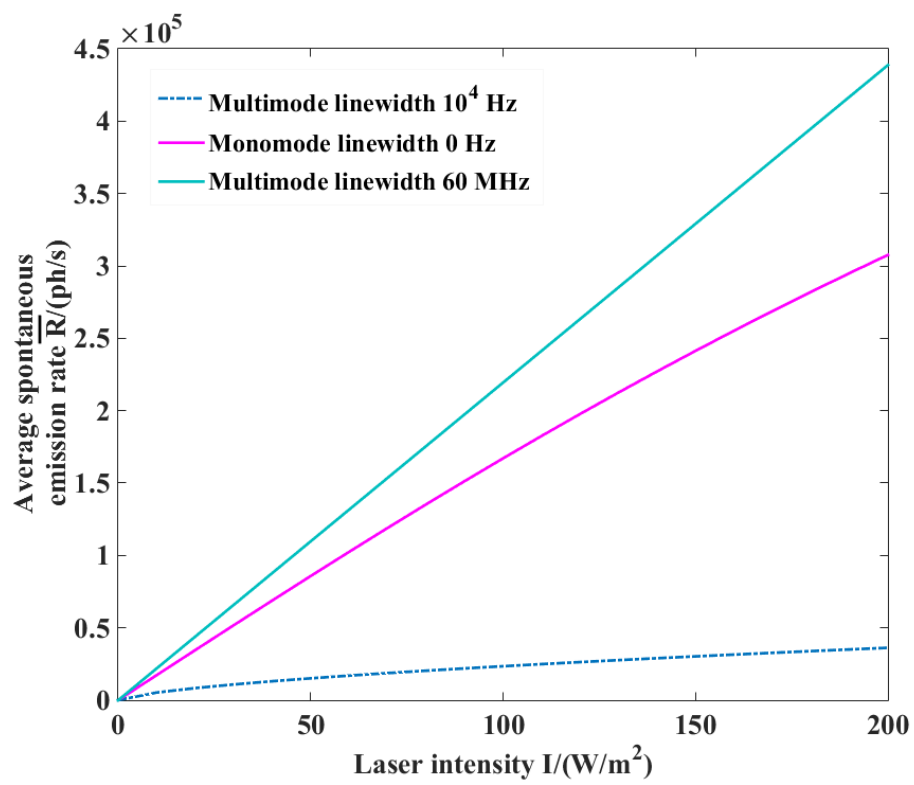

Figure 15. Average spontaneous emission rates vs. intensity at linewidth $0,1,0.01 \mathrm{MHz}$. 
According to the results in Figure 15, when the linewidth of the three-mode CW laser is broadened to $60 \mathrm{MHz}$, the average spontaneous emission rates increase over 10 times at $\mathrm{I}=150 \mathrm{~W} / \mathrm{m}^{2}$ and exceed those values of the mono-mode laser at linewidth $0 \mathrm{MHz}$. Such a case greatly enhances the return photons of the sodium laser guide star. Thus, we produce a result that the linewidth broadening method is capable of improving the recoil effects of the sodium laser guide star in the case of the multi-mode laser.

\section{Conclusions}

This article carefully investigates the effects of the linewidth broadening method on the recoil of the sodium laser guide star. The numerical results show that the linewidth broadening method increases the return photons of the sodium laser guide star. Moreover, for a laser over $50 \mathrm{~W}$, the return photons increase more than one time. Furthermore, this method will not make a difference in the spot sizes of the sodium laser guide star.

The optimal values of linewidth broadening are 1-100 MHz according to our simulations. This linewidth is capable of achieving wholly optimal average spontaneous emission rates. Our simulations reveal that the mono-mode CW laser at a linewidth less than $1 \mathrm{MHz}$ and more than $100 \mathrm{MHz}$ cannot obtain high average spontaneous emission rates. In order to obtain the best results, the laser linewidth should be modulated to be $1 \mathrm{MHz}$ or between 1 and $100 \mathrm{MHz}$. In addition, the sideband linewidth of re-pumping should be correspondingly broadened. Further analysis shows that the linewidth broadening method is capable of weakening the recoil effects of the sodium laser guide star for the multimode laser. A simulated result of the three-mode laser indicates that the average spontaneous emission rates of the sodium laser guide star significantly improve.

Several cases have proven that the linewidth broadening method is consistent with the actual situation. The current laser linewidth modulation method can provide a reference for laser linewidth broadening. We hope that our results are proven by experiments.

Author Contributions: Conceptualization, X.L. and X.Q.; methodology, X.L.; software, D.L.; validation, C.F. and H.Y.; formal analysis, R.H. and C.C.; investigation, X.L. and C.C.; writing-original draft preparation, X.L.; writing-review and editing, R.H.; visualization, X.L. All authors have read and agreed to the published version of the manuscript.

Funding: This work was supported by the Open Research Fund of State Key Laboratory of Pulsed Power Laser Technology (No. SKL2020KF06) and the key projects of science foundations of Anhui Education Department (Nos. KJ2017A401 and KJ2019A0619) and the domestic visiting program of West Anhui University (No. wxxygnfx2019003).

Institutional Review Board Statement: Not applicable.

Informed Consent Statement: Not applicable.

Data Availability Statement: Data are contained within this article.

Conflicts of Interest: The authors declare that they have no conflict of interest to report regarding the present study.

\section{References}

1. Jonas, H.; Jörg, G. The global mesospheric sodium layer observed by Odin/OSIR-IS in 2004-2009. J. Atmos. Solar-Terr. Phys. 2011, 73, 2221-2227.

2. Holzlölner, R.; Rochester, S.M.; Bonaccini, C.D.; Budker, D.; Higbie, J.M.; Hackenberg, W. Optimization of cw sodium laser guide star efficiency. Astron. Astrophys. 2010, 510, A20. [CrossRef]

3. Bian, Q.; Bo, Y.; Zuo, J.; Li, M.; Dong, R.; Deng, K.; Xu, Z. Investigation of return photons from sodium laser beacon excited by a 40-watt facility-class pulsed laser for adaptive optical telescope applications. Sci. Rep. 2018, 8, 9222. [CrossRef] [PubMed]

4. Hillman, P.D.; Drummond, J.D.; Denman, C.A.; Fugate, R.Q. Simple Model, including Recoil, for the Brightness of Sodium Guide Stars created from CW Single Frequency Fasors and Comparison to Measurements. In Proceedings of the Astronomical Telescopes and Instrumentation: Synergies Between Ground and Space, Marseille, France, 23 June 2008.

5. Telle, J.; Drummond, J.; Hillman, P.; Denman, C. Simulations of mesospheric sodium guidestar radiance. In Proceedings of the Lasers and Applications in Science and Engineering, San Jose, CA, USA, 19 January 2008. 
6. Pedreros Bustos, F.; Holzlöhner, R.; Rochester, S.; Bonaccini Calia, D.; Hellemeier, J.; Budk, D. Frequency chirped continuous-wave sodium laser guide stars: Modeling and optimization. J. Opt. Soc. Am. B 2020, 37, 1208-1218. [CrossRef]

7. Chamoun, J.; Digonnet, M. Aircraft-navigation-grade laser-driven FOG with Gaussian-noise phase modulation. Opt. Lett. 2017, 42, 1600-1603. [CrossRef] [PubMed]

8. Liu, X.; Qian, X.; Li, Y.; Rao, R. Calculation and analysis of the number of return photons from Sodium Laser Beacon excited by the long pulse laser with circular polarization. Chin. Phys. B 2014, 12, 240-248. [CrossRef]

9. Milonni, P.W.; Fugate, R.Q.; Telle, J.M. Analysis of measured photon returns from sodium beacons. Opt. Soc. Am. A 1998, 15, 218-233. [CrossRef]

10. Jeys, T.H. Development of a mesospheric sodium laser beacon for atmospheric adaptive optics. In Proceedings of the LEOS '90. IEEE Lasers and Electro-Optics Society Annual Meeting, Boston, MA, USA, 6-8 November 1990.

11. Mcclelland, J.J.; Kelley, M.H. Detailed look at aspects of optical pumping in sodium. Phys. Rev. A 1985, 31, 3704-3710. [CrossRef]

12. Milonni, P.W.; Thode, L.E. Theory of mesospheric sodium fluorescence excited by pulse trains. Appl. Opt. 1992, 31, 785-800. [CrossRef]

13. Pique, J.P.; Moldovan, I.C.; Fesquet, V. Concept for polychromatic laser guide stars: One-photon excitation of the 4 P3/2 level of a sodium atom. J. Opt. Soc. Am. A 2006, 23, 2817-2828. [CrossRef]

14. Morris, J.R. Efficient excitation of a mesospheric sodium laser guide star by intermediate -duration pulses. J. Opt. Soc. Am. A 1994, 11, 832-845. [CrossRef]

15. Milonni, P.W.; Fearn, H. Theory of continuous-wave excitation of the sodium beacon. J. Opt. Soc. Am. A 1999, 16, 2555-2566. [CrossRef]

16. Rodolphe, C.; Olivier, L.; Glen, H.; Bradley, C.; Kate, J. Experimental assessment of the matched filter for laser guide star wavefront sensing. Appl. Opt. 2009, 48, 1198-1211.

17. Liu, X.; Qian, X.; Zhu, W.; Rao, R.; Wei, X.; Lu, C. Characteristics of return photons from the polychromatic laser guide star excited by a long pulse laser with $330 \mathrm{~nm}$ wavelength. In Proceedings of the Conference on Laser Technology and Optoelectronics, Chengdu, China, 17-20 October 2019.

18. Moussaoui, N.; Holzlöhner, R.; Hackenberg, W.; Bonaccini, C.D. Dependence of sodium laser guide star photon return on the geomagnetic field. Astron. Astrophys. 2009, 501, 793-799. [CrossRef]

19. Drummond, J.; Novotny, S.; Denman, C.; Hillman, P.; Telle, J. The Sodium LGS Brightness Model over the SOR. In Proceedings of the Advanced Maui Optical and Space Surveillance Technologies Conference, Wailea, HI, USA, 12-15 September 2007.

20. Qian, X.; Zhu, W.; Rao, R.Z. Long-distance propagation of pseudo-partially coherent Gaussian Schell-model beams in atmospheric turbulence. Chin. Phys. B 2012, 21, 094202. [CrossRef]

21. Chen, H.; Ji, X.; Li, X.; Wang, T.; Zhao, Q.; Zhang, H. Energy focusability of annular beams propagating through atmospheric turbulence along a slanted path. Opt. Laser Technol. 2015, 71, 22-28. [CrossRef]

22. Jin, K.; Wei, K.; Feng, L.; Bo, Y.; Zuo, J.; Li, M.; Fu, C.; Dai, X.; Bian, Q.; Yao, J.; et al. Photon Return On-Sky Test of Pulsed Sodium Laser Guide Star with D-2b Repumping. Publ. Astron. Soc. Pac. 2015, 127, 749-756. [CrossRef]

23. Harding, C.M.; Johnston, R.A.; Lane, R.G. Fast Simulation of a Kolmogorov Phase Screen. Appl. Opt. 1999, 38, 2161-2170. [CrossRef]

24. Greenwood, D.P. Bandwidth specification for adaptive optics systems. J. Opt. Soc. Am. 1977, 67, 390-393. [CrossRef]

25. Ji, X.; Pu, Z.; Ji, G.; Jia, X. Regions of spreading of partially coherent beams propagating through non-kolmogorov turbulence. Opt. Commun. 2014, 322, 109-113. [CrossRef]

26. Chen, M.; Gao, T.; Hu, S.; Zeng, Q.; Liu, L.; Li, G. Simulating Non-Kolmogorov turbulence phase screens based on equivalent structure constant and its influence on simulations of beam propagation. Results Phys. 2017, 7, 3596-3602. [CrossRef]

27. Yan, H.X.; Li, S.S.; Zhang, D.L.; Chen, S. Numerical simulation of an adptive optcs system with laser propagation in the atmosphere. Appl. Opt. 2000, 38, 3023-3031. [CrossRef] [PubMed]

28. Rubio, J.A.; Belmonte, A.; Comerón, A. Numerical simulation of long-path spherical wave propagation in three-dimensional random media. Opt. Eng. 1999, 38, 1462-1469. [CrossRef]

29. Parenti, R.R.; Sasiela, R.J. Laser-guide-star systems for astronomical applications. J. Opt. Soc. Am. A 1994, 11, 288-309. [CrossRef]

30. Pennington, D.M. Laser Guided Adaptive Optics for High-Resolution Astronomy. In Proceedings of the Conference on Lasers and Electro-Optics, Long Beach, CA, USA, 19-22 May 2002.

31. Pique, J.P.; Farinotti, S. Efficient modeless laser for a mesospheric sodium laser guide star. J. Opt. Soc. Am. B 2003, 20, 2093-2101. [CrossRef]

32. Denman, C.A.; Drummond, J.D.; Hillman, P.D.; Steven, J.N.; Telle, J.M. Characteristics of sodium guidestars created by the 50-watt FASOR and first closed-loop AO results at the Starfire Optical Range. In Proceedings of the Advances in Adaptive Optics II, Orlando, FL, USA, 24-31 May 2006.

33. D'Orgeville, C.; Rigaut, F.J.; Ellerbroek, B.L. LGS AO photon return simulations and laser requirements for the Gemini LGS AO program. In Proceeding of the SPIE Astronomical Telescopes and Instrumentation, Adaptive Optical Systems Technology, Munich, Germany, 27 March 2000. 